\title{
Low complexity precoding matrix selection method for Long-Term Evolution systems
}

\author{
Chi-Min Li ${ }^{1}$, Wei-Wen Tsao ${ }^{1}$, and Pao-Jen Wang ${ }^{2 a)}$ \\ ${ }^{1}$ Department of Communications, Navigation and Control Engineering, National \\ Taiwan Ocean University, 2 Pei-Ning Road, Keelung City, 20224, Taiwan, R.O.C. \\ ${ }^{2}$ Department of Electrical Engineering, Ming Chi University of Technology, \\ 84 Gungjuan Road, Taishan District, New Taipei City, 24301, Taiwan, R.O.C.
}

a)pjwang@mail.mcut.edu.tw

\begin{abstract}
In a Long-Term Evolution system, a transmitter can appropriately transmit layered data streams via multiple antennas with the help of a precoding matrix. The precoding matrix can be selected using the feedback precoding matrix indicator information from the receiver. Different precoding matrixes will affect the system capacity and bit error rate performance dramatically. In this paper, we propose a low complexity precoding matrix selection method based on trace calculation of the MIMO channels' eigenvalue. The method will have almost the same capacity as the maximum mutual information (MI) method, as well as the low computational complexity advantage. Simulation results show that the proposed method requires less than $20 \%$ calculations needed by the maximum MI method.
\end{abstract}

Keywords: MIMO, LTE, Precoding Matrix Indicator (PMI)

Classification: Wireless Communication Technologies

\section{References}

[1] 3GPP TS 36.211 V12.3.0, Evolved Universal Terrestrial Radio Access (EUTRA), Physical channels and modulation (Release 12), Sept. 2014.

[2] S. Schwarz, C. Mehlfuhrer, and M. Rupp, "Calculation of the spatial preprocessing and link adaption feedback for 3GPP UMTS/LTE," Proc. 2010 6th Conferece on Wireless Advanced (WiAD), pp. 1-6, Jun. 2010. DOI:10.1109/ WIAD.2010.5544947

[3] W. Fu, F. Zhang, G. Yantao, and Z. Cui, "Research on the selection algorithm of precoding matrix in TDD LTE," Proc. 2011 IEEE 3rd International Conference on Communication Software and Networks (ICCSN), pp. 365-368, May 2011. DOI:10.1109/ICCSN.2011.6013613

[4] D. Tse and P. Viswanath, Fundamentals of Wireless Communications, Cambridge University Press, 2005. 


\section{Introduction}

Long-Term Evolution Advanced (LTE-A), which is defined by the 3rd Generation Partnership Project (3GPP), has been deployed widely as the fourth-generation (4G) wireless communication system owing to its high spectral utilization and throughput performance. The LTE-A system adopts the orthogonal frequency division multiple access (OFDMA) and multiple input multiple output (MIMO) techniques to achieve high-speed service for the served user equipment (UE).

To cope with time-varying fading variations of the wireless channels, the transmitter (Tx) usually uses feedback channels to report current channel state information (CSI) and adjusts transmit parameters such as modulation, code rate, spatial layer, and adopted precoding matrix. In the LTE system, CSI can be acquired at the Tx in terms of three primary parameters, i.e., rank indicator (RI), precoding matrix indicator (PMI), and channel quality indicator (CQI). These parameters can help the Tx to dynamically select the appropriate parameters for the signals to achieve better and reliable transmission. Therefore, deciding on appropriate and efficient selection of feedback information is an important issue for LTE systems.

Among the various pieces of feedback information, the precoding matrix serves to appropriately distribute the layered data streams transmitted by the multiple Tx antennas. Different precoding matrix selections will dramatically influence system capacity and system bit error rate (BER) performance. For the 3GPP LTE system, the two-transmit antennas, four-transmit antennas, and eight-transmit antennas precoding matrices are well-defined [1].

Besides, many literatures have provided methods for determining the appropriate PMI for LTE systems. In [2], the authors described a precoding matrix selection method based on mutual information (MI) maximization. This method has very good capacity performance; however, the associated computational complexity is high, especially because of the intensive signal to noise ratio (SNR) and MI calculations. In [3], the authors reported a low complexity precoding matrix selection method based on the maximization of the equivalent channel gains. In this paper, we propose a low complexity precoding matrix selection method involving maximization of the trace of the eigenvalue of the MIMO channels. The proposed method will have almost the same capacity ability as the maximum MI method [2], but it will be characterized by lower computational complexity compared with the literary methods. The proposed method and the compared literature methods are described in Section 2. Performance analysis of these methods, including system capacity and computational complexity, is reported in Section 3. The conclusions of this paper are summarized in Section 4.

\section{Method descriptions}

The signal model considered in this paper is shown in Fig. 1. The $N_{r} \times 1$ received signal vectors $y_{k}$ at the $k$ th subcarriers of the OFDM signal can be expressed as follows:

$$
y_{k}=H_{k} W_{i} x_{k}+n_{k}
$$


where $H_{k}$ is the $N_{r} \times N_{t}$ MIMO channel matrix; $N_{t}$ is the number of transmit antennas; $N_{r}$ is the number of receiving antennas; $W_{i}$ is the $N_{t} \times 1$ precoding matrix to be determined; $x_{k}$ is the $l \times 1$ transmitted signal vector, where $l$ is the spatial layer during transmission; and $n_{k}$ is the $N_{r} \times 1$ additive white Gaussian noise (AWGN). Assume that the spatial layer has been determined before the PMI selection. After equalization, the output signal is

$$
r_{k}=G_{k} y_{k}=G_{k} H_{k} W_{i} x_{k}+G_{k} n_{k}
$$

where $G_{k}$ is the equalizer compensation, which can be expressed by Eqs. (3) and (4) for the minimum mean square error (MMSE) and zero forcing (ZF) criteria, respectively [4].

$$
\begin{gathered}
G_{Z F}=\left[\left(H_{k} W_{i}\right)^{H}\left(H_{k} W_{i}\right)\right]^{-1}\left(H_{k} W_{i}\right)^{H} \\
G_{M M S E}=\left[\left(H_{k} W_{i}\right)^{H}\left(H_{k} W_{i}\right)+\left(\frac{N_{r} N_{0}}{E_{s}}\right) I\right]^{-1}\left(H_{k} W_{i}\right)^{H}
\end{gathered}
$$

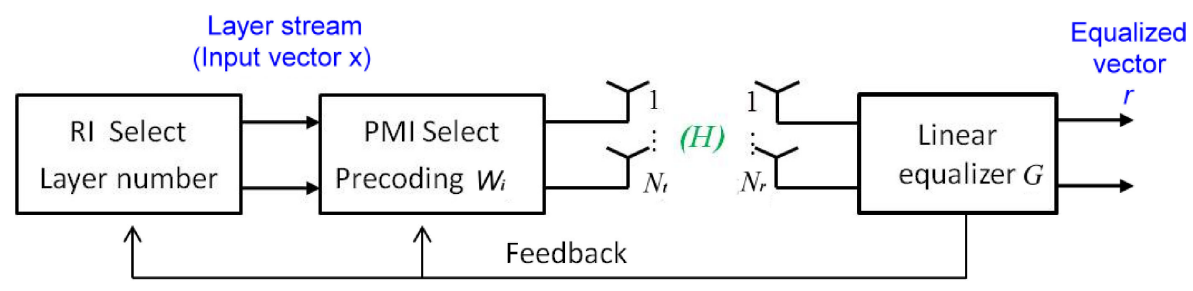

Fig. 1. Signal model.

\subsection{Maximum mutual information selection [2]}

In [2], the authors reported a precoding matrix selection method based on maximizing the Shannon capacity criteria of the transmission. The signal to interference and noise ratio (SINR) after the equalizer processing can be expressed as follows:

$$
\begin{gathered}
\operatorname{SINR}_{l, k}=\frac{\left|K_{k}(l, l)\right|^{2}}{\sum_{i \neq l}\left|K_{k}(l, i)\right|^{2}+\sigma_{n}^{2} \sum_{i} G_{k}(l, i)} \\
K_{k}=G_{k} H_{k} W_{i}
\end{gathered}
$$

where $k$ is the subcarrier index, and $l$ and $i$ are the indexes for the row and column, respectively. It is well-known that the channel capacity is the maximum of the MI between the transmitted signal and the equalized output. The maximum MI of a fading channel can be given by the Shannon theorem as follows:

$$
I_{k}=\sum_{l=1}^{L} \log _{2}\left(1+\operatorname{SINR}_{l, k}\right)
$$

where $L$ is the number of spatial layers of the LTE system. The precoding matrix can be selected using Eq. (8).

$$
W_{j}=\underset{W_{i} \in W}{\arg \max } \sum_{k=1}^{K} I_{k}\left(W_{i}\right)
$$

Clearly, many calculation steps are required to determine the SINR from $K_{k}$ and $G_{k}$ obtained using Eq. (5). Therefore, the same paper described a low complexity 
selection version as well by replacing Eq. (7) with Eq. (9) to avoid the SINR calculations and reduce computational complexity.

$$
I_{k}\left(W_{i}\right)=\log _{2} \operatorname{det}\left(I+\frac{1}{\sigma_{n}^{2}} W_{i}^{H} H_{k}^{H} H_{k} W_{i}\right)
$$

\subsection{SVD-based selection [3]}

In [3], the authors adopted singular value decomposition (SVD) for selecting the appropriate PMI. Let the SVD of the channel matrix be

$$
H=U D V^{H} .
$$

where $U$ and $V$ are the unitary matrixes with dimensions $N_{r} \times N_{r}$ and $N_{t} \times N_{t}$, respectively. $D$ is a diagonal matrix. Let the characteristic of the equalizer at $\mathrm{Rx}$ be $G_{k}=U^{H}$, and let the precoding matrix at $\mathrm{Tx}$ be $W_{i}=V$. Then, the received signal can be expressed as follows:

$$
Y=U^{H} H W_{i} X=U^{H} U D V^{H} V X=D X
$$

$X$ is the transmitted signal. Define an equivalent channel (EC) as follows:

$$
P_{\left(W_{i}\right)}=U^{H} H W_{i}=D V^{H} W_{i}
$$

Then, the suitable PMI for this method can be determined using Eq. (13):

$$
W_{j}=\underset{W_{i} \in W}{\arg \max }\left|\sum_{j=1}^{L} P_{\left(W_{i}\right)}(j, j)\right|
$$

$L$ is the number of spatial layers. Basically, the method tries to find a precoding matrix that can achieve the best equivalent channel gain during the ongoing MIMO transmission.

\subsection{Proposed PMI selection method}

In the proposed method, the channel capacity of the MIMO system can be expressed as follows:

$$
\begin{aligned}
C(H) & =\log _{2} \operatorname{det}\left(I_{N_{r}}+\frac{\rho}{N_{t}} H W_{i} W_{i}^{H} H^{H}\right) \\
& =\sum_{i=1}^{N_{r}} \log _{2}\left(1+\frac{\rho}{N_{t}} \lambda_{i}\right)
\end{aligned}
$$

where $\lambda_{i}$ is the eigenvalue of matrix $H H^{H}$. It can be easily shown that the eigenvalues share the following relation with the channel matrix:

$$
\operatorname{tr}\left(H W_{i} W_{i}^{H} H^{H}\right)=\sum_{i=1}^{N_{r}} \lambda_{i}
$$

where $\operatorname{tr}($.$) is the trace operator. The proposed method selects the precoding matrix$ according to Eq. (16) to ensure the maximum channel capacity for the MIMO transmission.

$$
W_{j}=\underset{W_{i} \in W}{\arg \max }\left\{\operatorname{tr}\left(H W_{i} W_{i}^{H} H^{H}\right)\right\}
$$


summation of the MIMO channels (Eqs. 15 and 16). It is shown in Section 3 that the proposed method has almost the same capacity performance as the maximum MI method, but with computational complexity lower than the other methods in the literature.

\section{Computer simulations}

In this section, the channel capacity performance and computational complexity of the proposed method are evaluated and compared with the corresponding values of other methods in the literature. In addition, it is assumed that the channel estimation can be acquired perfectly at the Rx. The MIMO channels are assumed to be identical, independent distributed complex Gaussian random variables. The antenna numbers at the Tx and Rx are 4 and 3, respectively. The MMSE equalizer is used in this simulation.

Fig. 2 shows the system capacity performance of different methods. From the results, the SVD-based method has the worst capacity performance, whereas the maximum MI method, its simplified version, and the proposed method have almost identical capacity performance. In Eq. (14), it is clear that the channel capacity depends mainly on the eigenvalues of the MIMO channel matrix. The proposed method uses the trace of the $H W_{i} W_{i}^{H} H^{H}$ which is directly related to the eigenvalues in Eq. (15) to select the precoding matrix. For the SVD method, it has a worse result than the other methods since that it adopts the equivalent channel to conduct the precoding matrix selection rather than the eigenvalues of the channel.

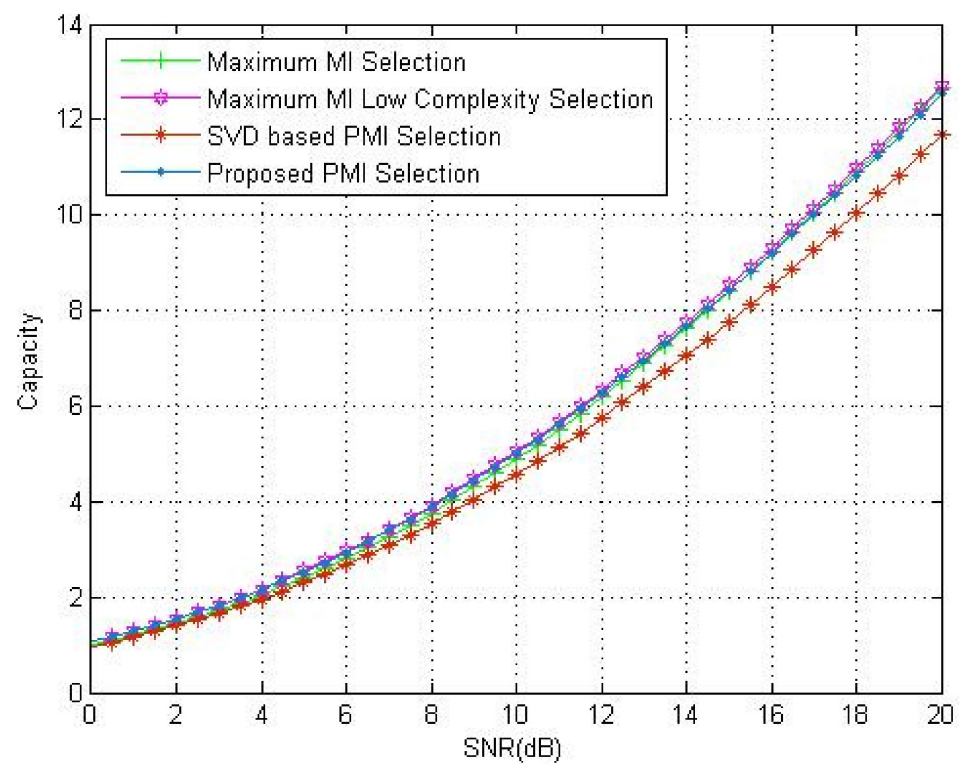

Fig. 2. Capacity performance.

However, the maximum MI method is plagued with high computational complexity, even the simplified version, which is further analyzed in the following text. The major contribution of the proposed method is the large reduction in computational complexity during PMI selection. Let $l=\min \left(N_{t}, N_{r}\right)$. Table I lists the computational complexity values of the methods compared herein. If we insert 
the simulation parameters in the calculations and set the calculations required by the maximum MI method to $100 \%$, the proposed method needs only $19.5 \%$ of the calculations used by the maximum MI method. The SVD method has almost the same low complexity advantage. However, the proposed method has better capacity performance than the SVD method, as shown in Fig. 2. Therefore, when considering both the capacity performance and the computational complexity in a practical implementation, the proposed method will be the best choice among the methods discussed herein.

Table I. Analysis of the computational complexity

\begin{tabular}{|c|c|c|}
\hline Computational complexity & \multicolumn{2}{|c|}{ Example } \\
\hline Maximum mutual information selection & \multirow[b]{2}{*}{9088} & \multirow[b]{2}{*}{$100 \%$} \\
\hline $\begin{array}{l}\sum_{l=1}^{L}\left\{N_{r} l N_{t}+N_{r} l\left(N_{t}-1\right)+l^{2} N_{r}+l^{2}\left(N_{r}-1\right)+l^{3}+l^{2}+l\right. \\
\left.\quad+l^{2} N_{r}+l N_{r}(l-1)+l^{2} N_{r}+l^{2}\left(N_{r}-1\right)+l+2(l-1)\right\}\end{array}$ & & \\
\hline Low complexity maximum mutual information selection & \multirow{2}{*}{2096} & \multirow{2}{*}{$23.1 \%$} \\
\hline$\left\{N_{r} l N_{t}+N_{r} l\left(N_{t}-1\right)+l^{2} N_{r}+l^{2}\left(N_{r}-1\right)+2 l^{2}+2 l-1\right\}$ & & \\
\hline SVD-based selection & \multirow{2}{*}{1776} & \multirow{2}{*}{$19.5 \%$} \\
\hline$\left\{N_{r} l N_{t}+N_{r} l\left(N_{t}-1\right)+N_{r}^{2} l+N_{r} l\left(N_{r}-1\right)+N_{r}\right\}$ & & \\
\hline Proposed selection & \multirow{2}{*}{1776} & \multirow{2}{*}{$19.5 \%$} \\
\hline$\left\{N_{r} l N_{t}+N_{r} l\left(N_{t}-1\right)+l^{2} N_{r}+l^{2}\left(N_{r}-1\right)+N_{r}\right\}$ & & \\
\hline
\end{tabular}

\section{Conclusions}

In this paper, we proposed a low complexity precoding matrix selection method for LTE systems. Without loss of any channel capacity, the proposed method offers the advantage of avoiding intensive PMI calculations at the Rx. Based on the simulation results, the proposed method requires less than $20 \%$ of the calculations used by the maximum MI method. Consequently, Rx can more speedily inform the Tx via the feedback channels, thus reducing the impact of the time delay due to the time-varying fading variations of the MIMO channels. 